\title{
A Comparative Analysis of Monofunctional Biosynthetic Peptidoglycan Transglycosylase (MBPT) from Pathogenic and Non-pathogenic Bacteria
}

\author{
Andrew T. Baker, Natsumi Takahashi and \\ Sathees B. Chandra*
}

Department of Biological, Chemical and Physical Sciences, Roosevelt University, Chicago, USA

\begin{abstract}
Monofunctional biosynthetic peptidoglycan transglycosylase (MBPT) catalyzes the formation of the glycan chain in bacterial cell walls from peptidoglycan subunits: N-acetylglucosamine (NAG) and acetylmuramic acid (NAM). Bifunctional glycosyltransferases such as the penicillin binding protein (PBP) have peptidoglycan glycosyltransferase (PGT) on their $C$ terminal end which links together the peptidoglycan subunits while transpeptidase (TP) on the $N$ terminal end cross-links the peptide moieties on the NAM monosaccharide of the peptide subunits to create the bacterial cell wall. The singular function of MBPT resembles the $C$ terminal end of PBP as it too contains and utilizes a similar PGT domain. In this article we analyzed the infectious and non infectious protein sequences of MBPT from 31 different strains of bacteria using a variety of bioinformatic tools. Motif analysis, dot-plot comparison, and phylogenetic analysis identified a number of significant differences between infectious and non-infectious protein sequences. In this paper we have made an attempt to explain, analyze and discuss these differences from an evolutionary perspective. The results of our sequence analysis may open the door for utilizing MBPT as a new target to fight a variety of infectious bacteria.
\end{abstract}

Keywords: peptidoglycan, infectious, pathogenicity, bacterial cell wall, penicillin

\section{Introduction}

Monofunctional biosynthetic peptidoglycan transglycosylase is a member of Glycosyltransferase Family 51 and present in over 3000 bacterial species as well as three

*Corresponding author: E-mail schandra@roosevelt.edu Tel +1-847-619-7968, Fax +1-847-619-8555 Accepted 4 June 2010 eukaryotic species: moss, green algae, and ameboid (Sauvage et al., 2008). As its name suggests, the protein is involved in the process of peptidoglycan synthesis by catalyzing the formation of glycan chains (Yuan et al., 2007). The Glycosyltransferase, GT, family can be divided into two categories: monofunctional GT and bifunctional GT which has a glycosyltransferase domain as well as transpeptidase domain (Terrak et al., 2008). The bifunctional GT class has been thoroughly studied as a target of antibiotics as penicillin is known to bind to the transpeptidase domain of the bifunctional GT or the penicillin-binding protein (Berardino, 1996; Barrett, 2007). Monofunctional biosynthetic peptidoglycan transglycosylase polymerizes disaccharide units to form glycan chains, which are cross-linked by transpeptidase to build the peptidoglycan walls of the bacterial cell (Berardino et al., 1996; Terrak et al., 2008).

MBPT catalyzes the formation of peptidoglycan subunits which consist of two monosaccharides: $\mathrm{N}$-acetylglucosamine (NAG or GlcNAc) and $\mathrm{N}$-acetylmuramic acid (NAM or MurNAc), that are connected by a $\beta(1,4)$ glycosydic bond (Terrak et al., 2008) (Fig. 1). The NAM monosaccharide has two different peptide moieties which are cross-linked by the transpeptidase enzyme on the $C$ terminal end of the PBP enzyme or by a monofunctional transpeptidase protein such as DD-transpeptidase, DD-carboxypeptidase, and DD-endopeptidase (Ghuysen et al., 1998; Yuan et al., 2007). The NAM peptide chain containing D-alanine, D-glutamic acid, and meso-diaminopimelic acid is present in the thinner cell walled, gram negative bacteria such as Hemophilus influenza. Gram positive bacteria such as Staphylococcus aureus have a thicker cell wall as the peptide sequence is: L-alanine, D-glutamine, L-lysine, and D-alanine. MBPT links the nucleophilic hydroxyl group on the fourth carbon of the NAG moiety to the electrophilic carbon with the protruding diphospholipid chain of the NAM moiety (Yuan et al., 2007). An over expression of the MBPT gene, $m g t$, causes an increase in the formation of the peptidoglycan subunits, in vitro, as further work can be done to analyze the precise lengths of the glycan chain created by monofunctional and bifunctional transpeptidase which may infer further differences between infectious and non infectious bacteria (Di Berardino et al., 1996). The generation of MBPT from the $m g t$ gene also gives MBPT its own identity separate from the bi- 


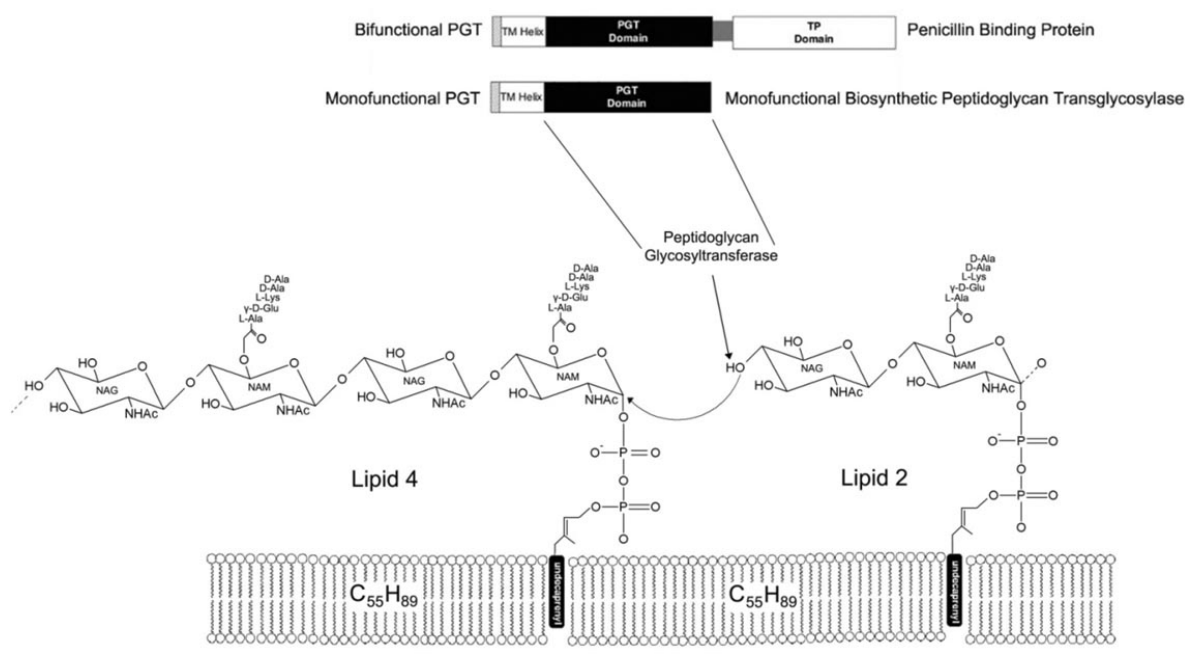

Fig. 1. Monofunctional Biosynthetic peptidoglycan transglycosylase (MBPT), also known as Peptidoglycan Glycosyltransferase, acts as an inducer of polymerization to produce the long carbohydrate chains of the bacterial cell wall. MBPT is a catalyst which joins the diasaccharide subunits (NAG-NAM) of membrane bound Lipid 2 intermediates to create elongated Lipid peptidoglycan subunits such as Lipid 4, 6, 8, to form the carbohydrate chain. Protein sequences of MBPT and PBP, penicillin binding protein, are presented above the mechanism to demonstrate the similarity between the monofunctional and bifunctional protein sequences and their usage of the PGT domain.

functional PBP which is transcribed from the $p b p$ gene even though both enzymes share a great deal of homology in their respective PGT domains (Barrett, 2007; DiBerardi et al., 1996; Paik et al., 1997; Spratt et al., 2006). Bioinformatic analysis of the monofunctional transpeptidase protein may provide further insight into the actions of infectious bacteria especially in comparison to MBPT and PBP. The precise role of each peptidoglycan synthesizing enzyme is still unknown as it is possible that the biosynthesis, repair, and upkeep of the cell wall is so vital that each enzyme may be called upon for a different reason be it synthesizing a new cell wall, repairing the cell wall, or fixing mistakes in the structure of the cell wall all of which are similar to the role of polymerases in DNA construction. Though the two proteins have similar roles, structures, and domains (PGT), MBPT is present in a great many more bacteria than PBP with a wide variety of habitats as well as forms of infection suggesting that MBPT may play a key role in the biosynthesis of the cell wall in a majority of bacteria and a new source of differentiation in the pathology of bacteria.

Bacterial pathogenicity has been one of the highly studied fields for obvious reasons and has lead to the identification of a variety of virulence factors such as adhesion molecules, metabolic enzymes, and toxins, which take advantage of a host's resources to allow the infectious bacteria to multiply and spread (Yang et al.,
2008). The degree of infection and its aggressiveness can be defined by the infectious bacteria' $s$ virulence which gages the potency of an infectious agent based on its ability to invade the host's system and induce disease. An infectious agent has to be able to penetrate the host's immune system and find an area in the host to multiply which is predicated on the infectious bacteria's mode of conveyance as well as the makeup of the cell's surface. The role of PBP has been deeply studied as it has been traditionally targeted by a variety of antibiotics giving it the name, penicillin binding protein (Suginaka et al., 2007). A number of sequenced genomes from a variety of bacterial species indicate that a majority of bacterial species have acquired genes via horizontal gene transfer which allows them to be better suited to survive in certain environmental conditions or niches (Schmidt and Hensel, 2004). Horizontal gene transfer can be achieved through a variety of mechanisms including natural transformation (Schmidt and Hensel, 2004), an exchange of plasmids and pathogenicity islands by way of conjugation (Schierack et al., 2006) as well as transduction by bacteriophages (Wagner and Waldor, 2002). Evolutionary differences between infectious and non infectious bacteria are apparent in their protein sequences as the two factions continue to separate from one another and exploit new environments for species proliferation. The purpose of this article is to analyze MBPT sequences derived from infectious and non 
infectious microbial organisms using a variety of bioinformatic tools. Here, we hypothesize that the sequence and the function of MBPT differs significantly between the two groups. The differences in the protein sequence may play a decisive role in defining the pathogenicity of a microbe.

\section{Methods}

The sequences for analysis were obtained from the NCBI website (http://www.ncbi.nlm.nih.gov/). Thirty one sequences were carefully selected based on their size, function, and organism origin. The selected sequences include fifteen sequences from bacterial species (fourteen gamma proteobacteria and one alpha proteobacteria) that are known to be infectious in humans and sixteen sequences from non infectious bacterial species (nine alpha proteobacteria and six beta proteobacteria). MBPT was chosen because of its occurrence in a number of non infectious as well as infectious bacteria from a wide variety of environments. The bacteria were selected based on the severity of pathogenicity as the most infectious diseases were found in the gamma pro- teobacteria family and represented in the infectious bacteria chosen for analysis.

Motifs were identified using the MEME Motif Discovery Tool at the default settings except for increasing the maximum number of motifs to ten (Bailey et al., 2006). The identification of similar motifs as well as the analysis of the phylogenic tree aided in the construction of the dot plots to further analyze the position of specific amino acid motifs in the selected protein sequences.

Dot matrices were constructed using the dotmatcher program in which the chosen sequences were compared to show similarity among the infectious bacteria and the non infectious bacteria (Landes et al., 1998). Similarity between the sequences was assessed by showing likeness through diagonal fragments in which a straight diagonal line exemplifies a high degree of similarity where as scattered diagonal fragments illustrate points of difference between the compared protein sequences. The sequences were assessed in the dot matcher program using a distance matrix, data matrix, and a chi-squared analysis to solely project the diagonal fragments of similarity between the axes of the graph. Dot-plot comparisons using the dotmatcher program

(a)

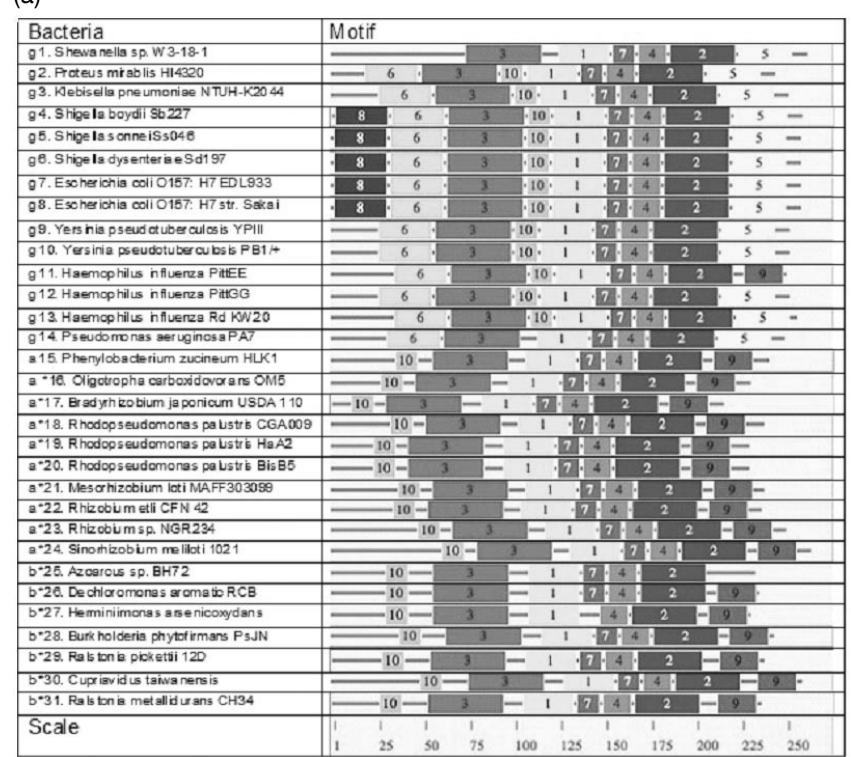

(b)
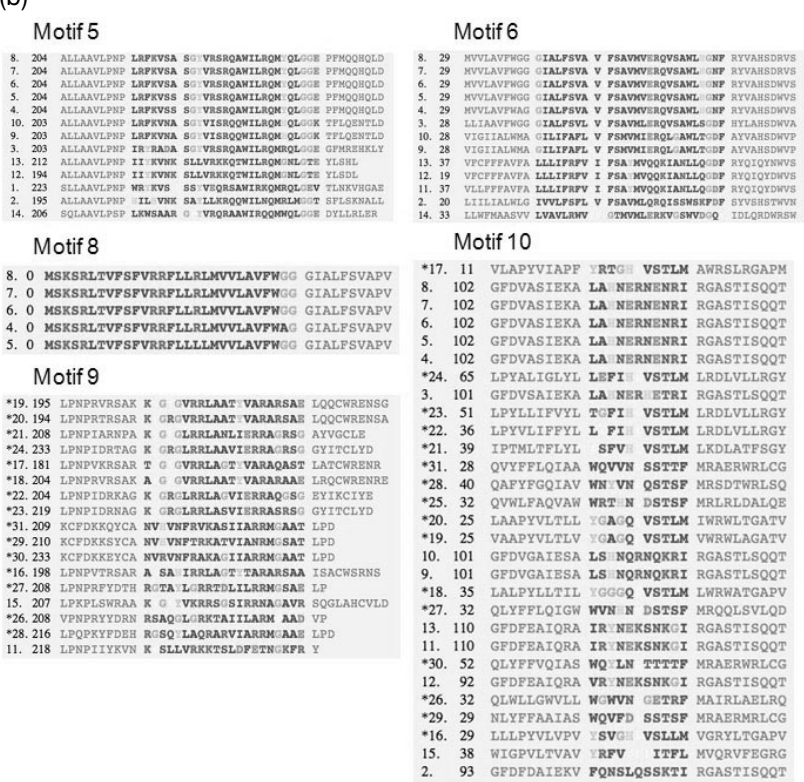

Fig. 2. (a) Placement of motifs within MBPT are listed in the table above. The numbers in the first column of the table identify the bacterial species and the letter preceding the number represents the bacteria's proteobacteria affiliation in such that: a represents alpha, $\mathrm{b}$ represents beta, and $\mathrm{g}$ represents gamma proteobacteria. The non infectious bacteria have an asterisk while the infectious bacteria do not. The numbers in this table represent each of the 31 bacteria species chosen for analysis for the (b) as well. (b) The sequences of motifs 5, 6, 8, 9, and 10 are presented in the figure above. The numbers to the left of each protein sequence correspond with the number and bacterial species in the table in (a). The second number is the length of the sequence in the MBPT enzyme followed by the sequence itself. For example: in motif 5 , the first bacterium is number 8, Escherichia coli 0157:H7 str. Sakai, has a length of 204 amino acids which are shown in the reading sequence. 
with a window size of 10 and a threshold of 23 were performed in order to examine the similarities between two sequences of infectious bacteria, noninfectious bacteria as well as between infectious and non-infectious bacteria. Further analysis was done to exemplify the differences between pathogenic and non pathogenic bacteria and to isolate and evaluate the subtle differences between the bacteria sequences.

Our sequences were aligned using Clustal X (Thompson et al., 1997). The phylogenic tree was constructed using the neighbor-joining method from the sequence alignment using PHYLIP (Felsenstein, 1989). The "Bootstrapping" method was performed on the phylogenic tree to assure the position of the clades as well as presenting evolutionary relationships between the infectious and non infectious bacteria. The tree was then viewed in TREEVIEW for further evaluation of the selected sequences (Page et al., 1996).

\section{Results}

When analyzing the MBPT motifs of infectious and non infectious bacteria it becomes apparent that there are several distinct differences between MBPT in infectious versus non infectious bacteria. The infectious bacteria share motifs 5 and 6 while the non infectious bacteria share domain 9 (Fig. 2). Motif 5 is present in sequences 1 (Shewanella sp. W3-18-1) through 10 (Yersinia pseudotuberculosis $P B 1 /+$ ) as well as sequence 12 (Haemophilus influenza PittGG) through 14 (Pseudomonas aeruginosa PA7) which are all members of the gamma proteobacteria family. Motif 5 is not present in the sequences of 11 (Haemophilus influenza PittEE) from the gamma proteobacteria family and 15 (Phenylobacterium zucineum HLK1) from the alpha proteobacteria family and is also missing from the non infectious bacteria group marked with an $\left(^{*}\right)$. Motif 9 is present in sequences 11 and 15 as well as all of the non infectious bacteria *16 (Oligotropha carboxidovorans OM5) through *31 (Ralstonia metallidurans $\mathrm{CH} 34$ ) with the exception of *25 (Azoarcus sp. BH72). Motif 6 is in all of the infectious bacteria except for 1 (Shewanella $s p$. W3-18-1) and 15 (Phenylobacterium zucineum HLK1) as well as being absent in all of the non infectious bacteria. Motif 8 exists only in the bacteria from two different genuses: Shigella and Escherichia as these two bacteria are closely related.

The dot plots show the degree of similarity between specified protein sequences of infectious and non infectious bacteria (Fig. 3). The dot plots comparing non infectious to infectious strains of bacteria enable specific points of the protein structure to be identified and analyzed. The data produced by the dot plots reinforces the minute differences found between specified protein sequences and can be used for further analysis of different amino acid residues among two different species of bacteria. The dot plots comparing non infectious to infectious bacteria underline the motifs that are conserved between the two types of bacteria such as motifs $1,2,3,4$, and 7 while reinforcing the motifs within specific domains that are not shared: $6,5,9$, and 8 .

As hypothesized, the phylogenic tree analysis identified two distinct clades: one aligning the infectious bacteria and the other aligning the non infectious bacteria (Fig. 4). The non- infectious bacteria are divided between alpha and beta classes of proteobacteria as al-
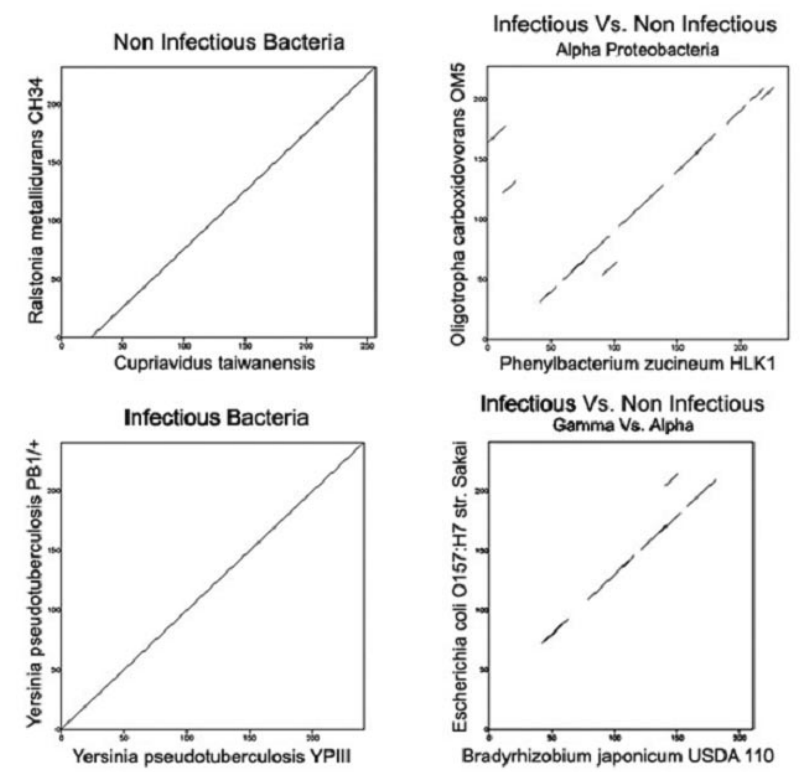

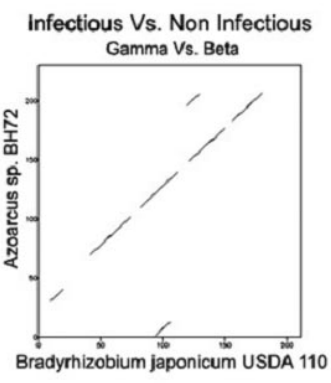

Fig. 3. The dot plot comparisons show the amount of dissimilarity between infectious and non infectious bacteria when an MBPT protein sequence from infectious and non infectious bacteria are compared to one another. Infectious and non infectious dot plot comparisons between two infectious MBPT sequences and two non infectious bacteria MBPT sequences are shown on the far left as a reference to the similarity within the two groups of bacteria. 


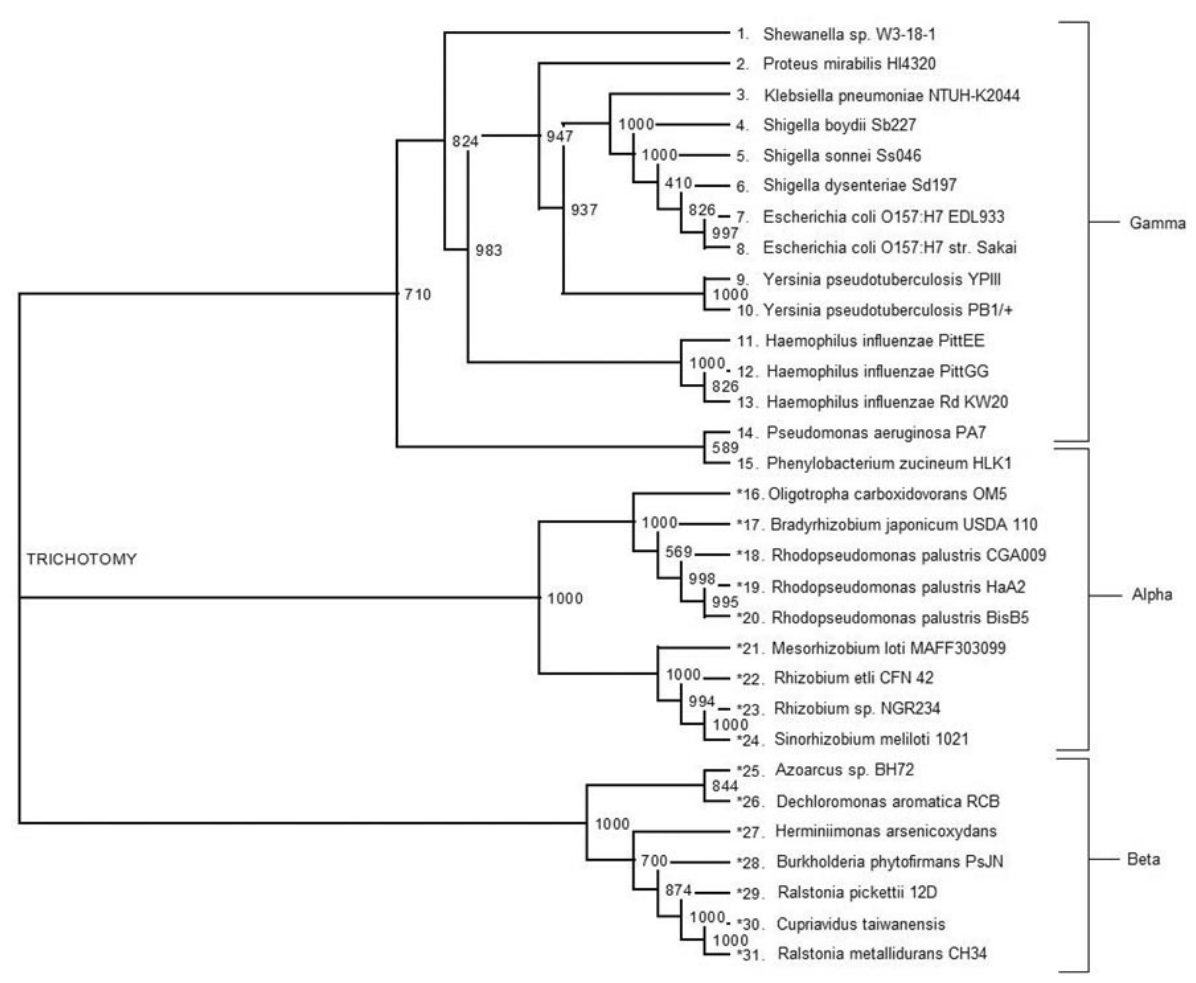

Fig. 4. A phylogenic tree constructed from the infectious and non-infectious bacterial sequences. The non infectious bacteria have an asterisk while the infectious bacteria do not. The scores are present to show the amount of relation between specific bacterial species. The bacteria are also grouped into their proteobacteria class, Gamma, Alpha, and Beta which is listed on the right for reference. The accession numbers for each bacterial species are as follows with the accession number in parentheses: Shewanella sp. W3-18-1 (YP_962288.1), Proteus mirabilis HI4320 (YP_002153355.1), Klebsiella pneumoniae NTUH-K2044 (YP_002921459.1), Shigella boydii Sb227 (YP_409502.1), Shigella sonnei Ss046 (YP_312164.1), Shigella dysenteriae Sd197 (YP_404871.1), Escherichia coli O157:H7 EDL933 (NP_289782.1), Escherichia coli O157:H7 str. Saka। (NP_312114.1), Yersinia pseudotuberculosis YPIII (YP_001719291.1), Yersinia pseudotuberculosis PB1/+ (YP_001874092.1), Haemophilus influenza PittEE (YP_001291277.1), Haemophilus influenza PittGG (YP_001292770.1), Haemophilus influenza Ro KW20 (NP_438991.2), Pseudomonas aeruginosa PA7 (YP_001345872.1), Phenylobacterium zucineum HLK1 (YP_002129110.1), Oligotropha carboxidovorans OM5 (YP_002287131.1), Bradyrhizobium japonicum USDA 110 (NP_767587.1), Rhodopseudomonas palustris CGA009 (YP_567451.1), Rhodopseudomonas palustris HaA2 (YP_484137.1), Rhodopseudomonas palustris BisB5 (YP_567451.1), Mesorhizobium loti MAFF303099 (NP_104813.1), Rhizobium etli CFN 42 (YP_471483.1), Rhizobium sp. NGR234 (YP_002827759.1), Sinorhizobium meliloti 1021 (NP_387372.1), Azoarcus sp. BH72 (YP_932210.1), Dechloromonas aromatica RCB (YP_287104.1), Herminiimonas arsenicoxydans (YP_001100990.1), Burkholderia phytofirmans PSJN (YP_001897032.1), Ralstonia pickettii 12D (YP_002982553.1), Cupriavidus taiwanensis (YP_002006627.1), Ralstonia metallidurans CH34 (YP_585194.1).

most all of the infectious bacteria are in the gamma proteobacteria family except for Phenylobacterium zucineum $H L K 1$, an alpha proteobacteria. The lower scores of some of the infectious bacteria like Shigella dysenteriae Sd197, Pseudomonas aeruginosa PA7, and Phenylobacterium zucineum HLK1 show some evolutional and sequential distance between these species of bacteria and the other species of infectious bacteria yet are able to remain within the infectious grouping. The bacteria Rhodopseudomonas palustris CGA009 exhibits some phylogenetic distance by its lower score while remaining in the non infectious group of bacteria. The evolutionary distance of bacteria in the phylogenic the tree shows adaptation of these bacteria to new niches that others are not able to propagate in and these differences become apparent in the sequence of MBPT for the particular bacteria.

\section{Discussion}

The phylogenic analysis of MBPT shows a definite separation in the protein sequence between a variety of in- 
fectious and non infectious bacteria. The formation of the phylogenic tree shows the possibility that infectious bacteria may have evolved from non infectious bacteria due to the separation of the two types of bacteria (Maurelli, 2006). An analysis of the bacterial motifs shows particular differences among the infectious and non infectious bacteria including the presence of motifs 5,6 , and 8 in the infectious bacteria. Absence and movement of motifs 9 and 10 are noted among the infectious and non infectious bacteria and may also glean more insight into the pathogenicity of bacteria. Motifs 1 , 2 , and 3 are recognized as being structurally required by all of the bacteria. The movement of motif 10 is evident in our motif analysis and further investigation may give us a clue as to why it is prominently in one position in the infectious bacteria and another in the non infectious bacteria. The Phenylobacterium zucineum HLK1 bacteria not only lacks the traditional motifs for infection but also has motif 10 arranged on the outside of the protein sequence in mimicry of a non infectious bacteria. The fact that an infectious bacteria can imitate a non infectious bacteria is concerning but the only clear anomaly in this study. The changes between specific amino acids in the MBPT sequences reinforces evolutionary changes between infectious and non infectious bacteria which enable the infectious bacteria to take advantage of new roles for further propagation of their species that the non infectious bacteria are unable to assume. Comparison of motif organization, amino acid sequences, dot plots and the phylogenic tree can isolate particular points of divergence between the infectious and non infectious bacterial sequences that may be exploited as a new source to fight infection. Further sequential, biochemical, and evolutionary analysis may continue to show details found in the analysis presented here for a novel, applicable treatment to a wide variety of infectious bacteria.

The bioinformatic analysis of Monofunctional Biosynthetic Peptidoglycan Transglycosylase presented here shows a clear distinction in the protein sequence between the infectious and non infectious bacteria. Further analysis can be done to create a viable application to fight a multitude of bacterial infection using MBPT. An analysis of the role of MBPT in cell division may further shed some light on its cellular role as well as its ability to evade antibiotics, like $\beta$-lactam, which inhibit the bifunctional transpeptidase activity of PBP (Di Berardino et al., 1996). A comparison of the monofunctional and bifunctional protein sequences may produce further insight in the pathogenicity of these cell wall building proteins. The analysis presented here provides a basis on which further research for utilizing MBPT as a new target for inhibiting bacterial infection can be built upon.

\section{References}

Bailey, T.L., Williams, N., Misleh, C., and Li, W.W. (2006). MEME: discovering and analyzing DNA and protein sequence motifs. Nucl. Acids Res. 34, W369-373.

Barrett, D., Wang, T.S.A., Yuan, Y., Zhang, Y., Kahne, D., and Walker, S. (2007). Analysis of glycan polymers produced by peptidoglycan glycosyltransferases. J. Biol. Chem. 282, 31964-31971.

Di Berardino, M., Dijkstra, A., Stuber, D., Keck, W., and Gubler, M. (1996). The monofunctional glycosytransferase of Escherichia coli is a member of a new class of peptidoglycan-synthesising enzymes. FEBS Letters 392, 184-188.

Felsenstein, J. (1989). PHYLIP Phylogeny Inference Package. Cladistics 5, 164-166.

Goffin, C., and Ghuysen, J.M. (1998). Multimodular penicillin-binding proteins: an enigmatic family of orthologs and paralogs. Microbiol. Mol. Biol. Rev. 62, 1079-1093.

Landes, C., Henaut, A., and Risler, J. (1998). Dot-Plot comparison by multivariate analysis (DOCMA): A tool for classifying protein sequences. Bioinformatics 9, 191-196.

Maurelli, A. (2006). Black holes, antivirulence genes, and gene inactivation in the evolution of bacterial pathogens. FEMS Microbiol. Lett. 267, 1-8.

Page, R. (1996). TREEVIEW: An application to display phylogenetic trees on personal computers. Computer Applications in the Biosciences 12, 357-358.

Paik, J., Jendrossek, D., and Hakenbeck, R. (1997). A putative monofunctional glycosyltransferase is expressed in Ralstonia eutropha. J. Bacteriol. 179, 4061-4065.

Rice, P., Longden, I. (2000). Emboss: the European Molecular Open Software Suite. Trends in Genetics 16, 276-277.

Sauvage, E., Kerff, F., Terrak, M., Ayala, J., and Charlier, P. (2008). The penicillin-binding proteins: structure and role in peptidoglycan biosynthesis. FEMS Microbiol. Rev. 32, 234-258.

Schierack, P., Steinruck, H., Kleta, S., and Vahjen, W. (2006). Virulence factor gene profiles of Escherichia coli isolates from clinically healthy pigs. Environmental Microbiology 72, 6680-6686.

Schmidt, $H_{\text {., }}$ and Hensel, M. (2004). Pathogenicity islands in bacterial pathogenesis. Clin. Microbiol. Rev. 17, 14-56.

Spratt, B.G. (1996). Monofunctional biosynthetic peptidoglycan transglycosylases. Mol. Microbiol. 19, 639-640.

Terrak, M., Sauvage, E., Derouaux, A., Dehareng, D., Bouhss, A., Breukink, E., Jeanjean, S., and Nguyen-Disteche, M. (2008). Importance of the conserved residues in the peptidoglycan glycosyltransferase module of the class A penicillin-binding protein $1 \mathrm{~b}$ of Escherichia coli. J. Biol. Chem. 283, 28464-28470.

Thompson, J., Gibson, T., Plewniak, F., Jeanmougin, F., and Higgins, D. (1997). The Clustal X windows interface: flexible strategies for multiple sequence alignment aided by quality analysis tools. Nucl. Acids Res. 25, 48764882.

Wagner, P., and Waldor, M.K. (2002). Bacteriophage control of bacterial virulence. Infection and Immunity 70, 39853993.

Yang, J., Chen, L., Sun, L., Yu, J., and Jin, Q. (2008). 
VFDB 2008 release: an enhanced web-based resource for comparative pathogenomics. Nucl. Acids Res. 36, D539-D542.

Yuan, Y., Barrett, D., Zhang, Y., Kahne, D., Sliz, P., and
Walker, S. (2007). Crystal structure of a peptidoglycan glycosyltransferase suggests a model for processive glycan chain synthesis. PNAS 104, 5348-5353. 